\title{
Population Research: Convenience Sampling Strategies
}

\author{
Samuel J. Stratton, MD, MPH $\mathbb{C}$
}

When conducting research that targets populations such as those living in a disaster affected area, it is often not possible to collect data or information from the entire population. This may occur when the members of the population cannot be individually identified or contacted, or the population is too large to allow for a census-based study (one in which each individual of the population is invited to participate in a study). In the circumstances where the entire population cannot participate in a study or the members of the target population cannot all be identified, sampling is employed to gather data that are presumed to be representative of that target population.

There are two primary categories of sampling strategies used in population research. First is probability sampling in which each member of the target population has an equal probability of being selected as a study participant. Common probability sampling methods include random sampling techniques such as simple, systematic, stratified, and cluster randomization. Important to note is that with probability (random) sampling techniques, there is less risk of bias in the results of the research, and most important, statistical methods such as optimal sample size and sampling error as well as precision of results can be determined. Probability sampling allows for conclusions and inferences about the target population of a study.

This discussion is directed to convenience sampling, a nonprobability form of sampling. Non-probability sampling methods are less objective than probability techniques and is a type of sampling in which the researcher uses sampling that does not provide for each member of a target population to participate in a study and rather the participants are selected by the researcher, are referred to the researcher, or self-select to participate in a study. There are multiple forms of non-probability sampling, the most common being purposeful sampling (participants are directly selected by the researcher), snowball recruiting (participants are referred to the researcher), and convenience sampling (the researcher announces the study and participants self-select if they wish to participate). Quota sampling is another form of non-probability sampling in which convenience sampling is layered onto a systematic population segmentation process (often done with "street" interviews in which people are profiled for characteristics that may fit the study objectives for participants).
Important is that non-probability sampling does not allow a researcher to extend the results of a study to a general population; rather, the results of non-probability sampling research can only apply to the participants in the research. Non-probability sampling research does not allow for determination of sampling error or precision of data relative to a target population. Empirical associations and causation that are generalizable outside the participant group of a non-probability-based study cannot be made.

Convenience sampling is non-probability sampling that is often used for clinical and qualitative research. This sampling technique often selects clinical cases or participants that are available around a location (such as hospital), medical records database, Internet site, or customer-membership list. Convenience sampling for qualitative research depends on the motivation of those who participate in the research. This introduces motivation bias into the study. Motivation to participate may depend upon the interest one has in the research topic, a wish to express a disgruntled point of view, or desire to support one's specific opinions.

In addition, there is risk of limited cases or poor participation rates for convenience samples. This raises the risk that significant data are not detected for case-based clinical studies. For survey or interview-based research, non-participant error is a concern because of risk of failure to obtain a balance of information for a research question. Non-participant error should be reported for qualitative research; for example, a response rate of $60 \%$ would generate a potential error of plus or minus $20 \%$ for any conclusion drawn from the research.

Convenience sampling is a common form of sampling found in population research and particularly in prehospital and disaster research. Convenience sampling is popular because it is not costly, not as time consuming as other sampling strategies, and simplistic. When used to generate a potential hypothesis or study objective, convenience sampling is useful. Despite the limitations of convenience sampling, there are steps that can be taken to improve credibility of this popular and simple method (Table 1).

As an overview, convenience sampling is a form of nonprobability sampling commonly used for population and clinical research. Convenience sampling is subject to multiple forms of bias and does allow for statistical assessment of sampling error or 


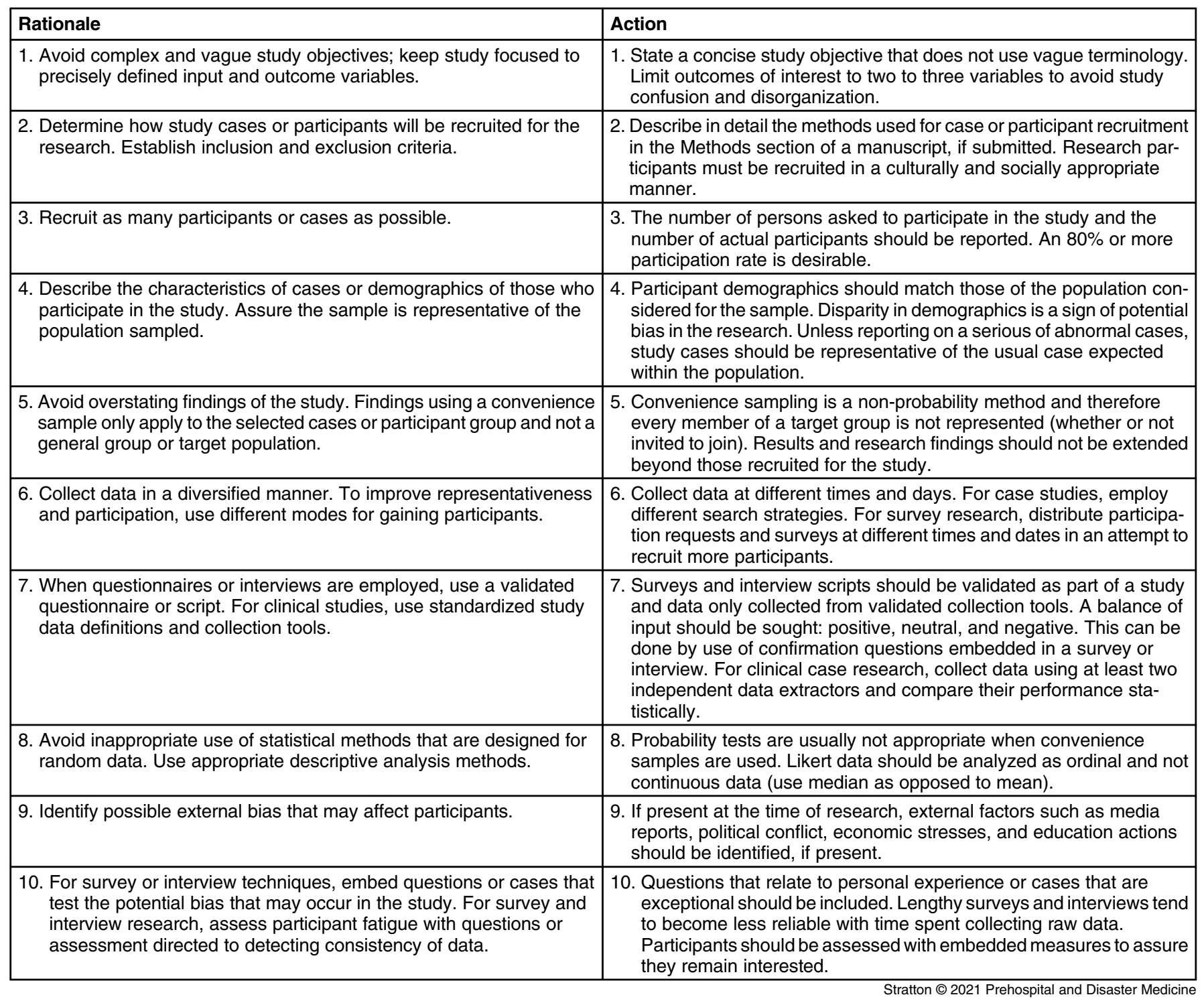

Table 1. Methods to Improve Dependability of Convenience Sampling

statistical validity. Analysis of convenience sample results can only be applied to the study participant group. Important is that associations and effects found with a convenience sample cannot be generalized to a target population. On the other hand, convenience sampling is less costly, quicker, and simpler than other forms of sampling. When no other sampling method is feasible, convenience sampling can be used to develop hypotheses and objectives for use in more rigorous research studies.

doi:10.1017/S1049023X21000649 